\title{
DISEÑO, APLICACIÓN Y EVALUACIÓN DE UNA PROPUESTA DIDÁCTICA PARA TRABAJAR LA ENSEÑANZA DE LOS CIRCUITOS ELÉCTRICOS EN TERCER CICLO DE EDUCACIÓN PRIMARIA
}

\author{
Autor:Javier Rodríguez Moreno \\ Tipo de tesis: Maestría \\ Director: Dr. Antonio de Pro Bueno \\ Departamento de Didáctica de las Ciencias Experimentales \\ Universidad Murcia \\ Programa: Máster Oficial en Investigación e Innovación en Educación Infantil y Educación Primaria \\ Fecha de presentación: junio de 2008
}

\section{RESUMEN}

El objetivo central del trabajo fue diseñar, aplicar y evaluar la unidad didáctica "Estudio de los circuitos eléctricos en la educación primaria”. Por ello, articulamos la investigación para dar respuesta a los siguientes interrogantes:

P.P.1: ¿Cuáles eran los conocimientos iniciales que tenía el alumnado sobre los circuitos eléctri$\cos$ ¿ ¿Cómo considerarlos en la planificación de la propuesta?

P.P.2: ¿Cómo se desarrollaron las actividades previstas? ¿Qué logros se obtuvieron, con qué dificultades se encontraron... en las actividades prácticas?

P.P.3: ¿Qué efectos produjo dicha propuesta en el alumnado en relación con el aprendizaje?

En primer lugar, revisamos qué decía el currículo respecto a estos contenidos y analizamos algunas contribuciones a la enseñanza y el aprendizaje de esta temática. Sobre la base de esta revisión y de nuestra experiencia, establecimos las hipótesis que se debían contrastar.

En relación con el diseño de la investigación, se trata del ensayo de una propuesta con pretest, seguimiento y postest. Los participantes eran alumnos de una clase de $5 .^{\circ}$ de EP de un CEIP de una población de Andalucía; se identificaron las características personales y académicas que delimitaban el contexto de la experiencia.

Para la planificación de la unidad didáctica, utilizamos un modelo basado en la realización de cinco tareas: análisis del contenido científico, análisis de los problemas de aprendizaje, determinación de los objeti- vos de aprendizaje, selección de estrategias de enseñanza y selección de estrategias de evaluación.

Para valorarla, utilizamos un pretest y un postest; en ambos casos, se trataba de cuestionarios de preguntas cotidianas. En relación con el seguimiento, hicimos una valoración de cada sesión mediante el diario del maestro; además, nos centramos en las tres actividades prácticas (estudio de circuitos con bombillas simples, en serie y en paralelo). En todos los casos, nos fijamos de forma especial en la presencia de los modelos alternativos de corriente (fluido, unipolar, interruptorfuente, atenuación...).

Respecto a los resultados del problema principal uno, podemos destacar que, a pesar de que el alumnado no había trabajado estos conocimientos en esta etapa, sus experiencias les permitían distinguir aparatos eléctricos de su entorno, conocer algunas de las normas de seguridad o identificar los elementos de un circuito sencillo. Esto puso de manifiesto una vez más que los niños de estas edades no son una "tabla rasa" a la hora de construir los conocimientos. De hecho, no solo los consideramos en el enfoque de los materiales, sino que utilizamos sus ideas continuamente en el propio proceso de construcción de sus aprendizajes. No obstante, también detectamos que no sentían, en general, la necesidad de utilizar un modelo de corriente en sus explicaciones y justificaciones.

Respecto a los resultados del problema principal dos, el desarrollo de la propuesta se ajustó a lo previsto en la planificación. El alumnado se implicó desde el principio y creemos que disfrutó aprendiendo. El trabajo en grupo favoreció el aprendizaje, probablemente porque era habitual en la clase. La incorporación de 
prácticas de laboratorio no solo los estimuló, sino que incrementó su interés. Resultó muy interesante el uso de los cuadernos de trabajo, en los que se recogían las preguntas que les planteábamos, sus respuestas y sus discusiones en grupo; en este sentido, se pudo observar una mejora sustantiva en la comunicación escrita. También nosotros disfrutamos a lo largo de nuestra experiencia.

En cuanto al seguimiento de algunas actividades prácticas, hemos de señalar que, en la referida al circuito simple, todos realizaron el montaje sin aparentes dificultades, comprobaron su funcionamiento e, incluso, no tuvieron problemas en la descripción e interpretación de lo realizado. En la práctica del circuito con bombillas en serie, también todos realizaron el montaje sin dificultad y contestaron adecuadamente a las preguntas referidas a la descripción de lo que iban observando; sin embargo, se apreciaron dificultades cuando debían explicar, interpretar, predecir..., hechos y fenómenos que habían realizado o provocado. En el circuito con bombillas en paralelo tuvimos peo- res resultados porque algunos tuvieron problemas en la realización del montaje; este hecho acrecentó las limitaciones en las cuestiones de interpretación, predicción y aplicación de los resultados.

Respecto a los resultados en el problema principal tres, el alumnado mantuvo los logros iniciales y mejoró las respuestas en relación con la interpretación del funcionamiento de los circuitos. No obstante, aparecieron en algunos casos y mantuvieron en otros los modelos alternativos de corriente eléctrica; estos condicionaron muchos de los razonamientos realizados. En cualquier caso, creemos que el interés y la motivación que se alcanzaron podrían ir más allá de los efectos en el aprendizaje a corto plazo que hemos detectado.

Globalmente nos parece una experiencia muy útil e interesante para la mejora de nuestro ejercicio profesional. Desde nuestro punto de vista, para una adecuada evolución y superación de los problemas que hemos debido afrontar, creemos necesario seguir investigando sobre las propuestas de enseñanza que realizamos todos los días en esta y otras etapas educativas.

\title{
EL DESEMPEÑO DE LAS COMPETENCIAS DE USO DE PRUEBAS Y MO- DELIZACIÓN EN UN PROBLEMA DE GESTIÓN DE RECURSOS MARINOS
}

\author{
Tesis doctoral \\ Autora: Beatriz Bravo Torija \\ Directora: María Pilar Jiménez Aleixandre \\ Departamento: Didáctica de las Ciencias Experimentales. Facultad de Ciencias de la Educación. Universi- \\ dad de Santiago de Compostela \\ Programa: Didáctica de las ciencias experimentales y de la matemática \\ Fecha: II de mayo del 2012
}

La propuesta de este estudio es examinar el desempeño de estudiantes de $4 .^{\circ}$ de ESO en las competencias de uso de pruebas y modelización durante la implementación de una secuencia de ecología. Este objetivo se divide en tres:

1. Examinar la capacidad de usar pruebas por el alumnado, caracterizando distintos niveles de complejidad en su desempeño e identificando las dificultades que experimentan.

2. Analizar los procesos de construcción y apropiación de las representaciones de las pirámides tróficas a través de los distintos mundos de conocimiento.
3. Analizar los procesos de aplicación y contextualización de modelos de ecología en el diseño de un plan de gestión de recursos a través de distintos estadios en la contextualización.

Los fundamentos teóricos proceden de distintos cuerpos de conocimiento en didáctica de las ciencias y se enmarcan fundamentalmente en tres: 1) argumentación y uso de pruebas; 2) modelización, como parte de la capacidad de explicar fenómenos científicamente, y 3) aprendizaje de la ecología y sus interrelaciones.

La metodología empleada es un estudio de caso múltiple. Los participantes son cuatro clases de $4 .{ }^{\circ}$ de ESO $(\mathrm{N}=66)$. 
La secuencia de ecología se divide en seis sesiones; en este trabajo nos centramos en dos de ellas: la construcción de las representaciones de las pirámides tróficas y la resolución de un problema de gestión de recursos marinos.

La toma de datos incluye grabaciones en audio y vídeo, producciones escritas de los estudiantes y notas de la investigadora.

Las herramientas de análisis y los resultados obtenidos para cada uno de los objetivos de investigación son:

- Caracterización del uso de pruebas: se elabora una progresión de aprendizaje a partir de la identificación y jerarquización de las operaciones que forman parte del uso de pruebas en la toma de decisiones, caracterizando cinco niveles de complejidad, y se aplica al discurso del alumnado. Los resultados obtenidos en cuanto a los informes escritos son que solo dos grupos se sitúan en el nivel superior; el resto se sitúa en niveles inferiores, aunque solo uno en el 1 . Ocho grupos alcanzan un nivel más alto de desempeño durante su discusión que en su informe final. Los resultados también muestran que la propuesta tiene potencial para examinar las dificultades de los estudiantes en el uso de pruebas, por ejemplo para integrar pruebas en justificaciones o para enmarcarlas en modelos teóricos.

- Construcción y apropiación de las pirámides tróficas: se elabora una herramienta basada en los mundos de conocimiento propuestos por Tiberghien (1994), a los que se añade el mundo de las representaciones, y se aplica al discurso del alumnado; el resultado es que el mundo de las representaciones es mayoritario en los dos procesos y las diferencias se encuentran en el tipo y número de relaciones que se establecen entre los mundos en cada proceso. También se identifican dificultades en la construcción de las pirámides, por ejemplo para atribuir un nivel trófico a cada organismo. Durante la apropiación solo los grupos que relacionan los tres mundos de conocimiento apelan al modelo del flujo de energía para explicar la forma triangular de las pirámides.

- Aplicación y contextualización de los modelos teóricos: se construye una herramienta para examinar el proceso de diseño del plan de gestión en términos de movimientos discursivos a través de diferentes estadios en la contextualización y se aplica al discurso del alumnado. Los resultados muestran que la categoría de mayor frecuencia es aplicar parcialmente un modelo, mayoritariamente el de mantenimiento de poblaciones, y en la categoría superior, aplicar los dos modelos, solo se sitúan cinco episodios. Para visualizar el proceso de diseño del plan de gestión, adaptamos una herramienta de Kelly y Takao (2002), las redes semánticas, mostrando que el diseño y la toma de decisiones no es lineal; todos los grupos, excepto uno, se mueven al menos entre dos categorías. La comparación de las estructuras discursivas de los grupos permite identificar similitudes, como el incremento en complejidad debido al apoyo del experto, y diferencias, por ejemplo en la interpretación del problema.

Las conclusiones que se derivan de los resultados obtenidos son:

En cuanto al primer objetivo: 1) se han caracterizado cinco niveles de complejidad en el desempeño en el uso de pruebas; 2) se ha elaborado una progresión de aprendizaje que muestra potencial para la identificación de las dificultades en esta práctica, y 3 ) el proceso de uso de pruebas y toma de decisiones de los pequeños grupos no es lineal.

En cuanto al segundo: 4) los mundos de conocimiento en los que se sitúa el discurso y las relaciones que se establecen están condicionados por el tipo de tareas que se realizan; 5) los estudiantes tienen dificultades para atribuir un nivel trófico a cada organismo y para decidir un tamaño para cada piso, y 6) el reconocimiento de que la disminución en los parámetros de biomasa y producción se debe a la disminución de energía está conectado con la capacidad de relacionar los tres mundos de conocimiento.

En cuanto al tercero: 7) la utilización del modelo de mantenimiento de poblaciones es mayor que la del modelo de eficiencia ecológica; 8) los movimientos discursivos a través de los diferentes estadios en la contextualización muestran que la toma de decisiones no es lineal, y 9) el apoyo de los expertos influye en el aumento de complejidad en el discurso del alumnado.

Una implicación que se deriva de este estudio es que para el desarrollo de las competencias es imprescindible involucrar a los estudiantes en estas prácticas científicas, por ejemplo si el objetivo es que apliquen un modelo, han de tener la oportunidad de construir representaciones y de reflexionar sobre sus significados. A su vez, también es útil para el profesorado, ya que facilita la identificación de dificultades y el diseño de tareas para ayudar a superarlas. 


\title{
CONCEPCIONES SOBRE EDUCACIÓN AMBIENTAL Y DESARROLLO PROFESIONAL DEL PROFESORADO DE CIENCIAS EXPERIMENTALES EN FORMACIÓN
}

\author{
Autor: Juan Diego Cardona Restrepo \\ Tipo de Tesis:Tesis doctoral \\ Directores: Roque Jiménez Pérez y Francisco Javier Perales Palacios \\ Departamento: Didáctica de las Ciencias y Filosofía \\ Universidad: Huelva \\ Programa: Didáctica de las ciencias \\ Fecha de presentación: I 3 de julio del 2012
}

En esta tesis doctoral se identifican los elementos del conocimiento didáctico y las creencias sobre la educación ambiental (EA) y el desarrollo profesional del profesorado de ciencias en formación. Para ello, nos planteamos los siguientes objetivos:

I. Elaborar un modelo teórico que exprese los diferentes componentes del conocimiento y las creencias del profesorado de ciencias respecto a la EA identificados en la literatura.

II. Utilizar este modelo para describir y explicar la progresión del conocimiento y las creencias respecto a la EA de un grupo de profesores de ciencias en formación inicial.

III. Determinar el efecto de la interacción social entre los profesores sobre la progresión en su conocimiento profesional de la EA.

IV. Establecer el impacto de un programa de formación en entornos colaborativos de investigación en EA sobre su desarrollo profesional.

V. Identificar los obstáculos y las dificultades que manifiestan los docentes para la integración de la EA y para su desarrollo profesional.

Partiendo de los paradigmas interpretativo y crítico-dialéctico, y bajo el enfoque de un estudio de caso, desarrollamos durante tres semestres académicos un programa de formación inicial relacionado con la investigación educativa en el contexto escolar sobre tópicos de la EA, en el que participaron doce maestros matriculados en las asignaturas Investigación monográfica y Proyecto pedagógico de la licenciatura en Educación Básica con énfasis en Ciencias Naturales y EA de la Universidad de Antioquia (Colombia). De entre ellos se seleccionaron tres maestros integrantes de uno de los equipos de trabajo, como objeto para el análisis en profundidad de sus producciones.

El investigador principal ejerce al mismo tiempo como tutor de los cursos mencionados. De acuerdo con la orientación metodológica, los datos fueron recopilados mediante cuestionarios, documentos escritos y una entrevista. Algunos de ellos en la primera fase del proceso, mientras que otros lo fueron al final. Esto con el propósito de comparar las concepciones de los maestros participantes en dos estadios diferentes y evaluar la incidencia del programa formativo sobre la evolución de su conocimiento didáctico y las creencias al respecto de la EA.

Se presta especial atención a dos factores que influyen decisivamente sobre el desarrollo profesional: $a$ ) la interacción social y $b$ ) la investigación en entornos colaborativos.

El modelo del conocimiento didáctico y las creencias sobre la EA ha sido construido a partir de las propuestas de Hashweh (2005) y Grossman (1990) y se compone de cuatro unidades referidas al conocimiento y las creencias: $a$ ) disciplinares, $b$ ) pedagógicas, $c$ ) curriculares y $d$ ) del contexto. Cada uno de estos elementos, en interacción dinámica, conforma el saber metadisciplinar que constituye la base fundamental del conocimiento profesional característico de los profesores.

En cuanto al desarrollo profesional, las variables han sido definidas en términos de los factores internos y externos que lo promueven u obstaculizan (Vázquez et al., 2010; Porlán et al., 2010; y Mellado, 2001). Son fuentes de este desarrollo, aparte de las ya mencionadas, la formación y experiencias previas de los participantes, la reflexión individual y grupal, así como el ejercicio de la profesión.

Analizando el contenido de las producciones de los maestros mediante los distintos instrumentos, se ha caracterizado la evolución de las concepciones de aquellos con respecto al conocimiento didáctico del contenido, ocupando un lugar destacado en las declaraciones individuales las variables del conocimiento y creencias disciplinares, por un lado, y el conocimiento 
y creencias sobre el contexto, por el otro. Caso contrario a los hallazgos encontrados en las construcciones grupales, en las que el conocimiento y las creencias pedagógicas se aprecian como las unidades de información preponderantes en las propuestas que atañen a la intervención educativa.

Al culminar el programa, los participantes demuestran disponer de conocimientos y competencias suficientes para construir propuestas interdisciplinares $y$ transversales de EA, hecho que se verifica en el tipo de problemas ambientales y el área que seleccionan cuando planean las estrategias de enseñanza. No obstante, son conscientes de sus debilidades y necesidades formativas, e igualmente reconocen que lo ideal sería la implementación de propuestas bajo los criterios y principios contemporáneos de la $\mathrm{EA}$, incorporando los saberes de todas las áreas escolares.
Con respecto al desarrollo profesional, factores internos tales como la motivación y el compromiso, la experiencia profesional previa y el conocimiento profesional son los que aparecen como determinantes para la promoción de aquel. Entre los factores externos que lo favorecen se tienen: la investigación en entornos colaborativos, la interacción social y las actividades y tareas del programa de formación.

Los maestros, al enfrentarse a situaciones de enseñanza y aprendizaje basadas en la investigación colaborativa de la propia práctica, mejoran su desempeño profesional y resuelven eficazmente los problemas pedagógico-didácticos, ofreciendo un nicho potencial para la evolución de sus concepciones y esquemas de actuación en el aula.

\title{
IMPLICACIONES CURRICULARES Y DIDÁCTICAS EN LA ENSEÑANZA DE LAS CIENCIAS DE LAS CONCEPCIONES SOBRE LA CIENCIA Y LA METODOLOGÍA EN ALUMNADO DE MAGISTERIO: EL TRABAJO POR PROYECTOS
}

\author{
Autora:Azucena Arias Correa \\ Dirección: Dra. María M. Álvarez Lires y Dr. Uxío Pérez Rodríguez \\ Lugar: Universidad de Vigo. Facultad de Ciencias de la Educación y del Deporte. Departamento de Didác- \\ ticas Especiales (Área de DCE). Campus de Pontevedra. \\ Programa: Investigación en didácticas especiales \\ Fecha: 18 de mayo del 2012
}

\section{Resumen:}

La investigación pretende desvelar concepciones previas del profesorado en formación inicial en materias relacionadas con las ciencias en diplomatura y grado, así como sus implicaciones para la consecución de competencias docentes que le permitan desarrollar y promover el acercamiento a las competencias básicas en su futuro alumnado de educación primaria e infantil, respectivamente, mediante el uso de métodos como el de proyectos.

\section{Marco teórico:}

Las investigaciones en DCE e informes internacionales como Rocard o ENCIENDE, sobre la enseñanza de las ciencias, aconsejan un cambio metodológico que posibilite una adecuada formación y la consecución de las competencias tecnocientíficas. Los proyectos que pueden responder a esta demanda contribuyen a formar una comunidad donde el alumnado trabaja en secuencias de aprendizaje con actividades que trasciendan un mero tratamiento teórico de las ciencias e incorporen experiencias, análisis, descripciones, argumentaciones, manejo de fuentes de información; trabaja en interacción; regula su aprendizaje y puede poner a prueba sus esquemas y modelos sobre el mundo sometiéndolos a un continuo proceso de explicitación, confrontación, experimentación y reestructuración para acercarlos a modelos propios de las ciencias.

Trabajar por proyectos requiere unas concepciones del profesorado que favorezcan el uso de metodologías innovadoras en las aulas. El profesorado en formación 
inicial posee ideas previas, creencias, mitos e imágenes sobre las ciencias y su enseñanza y es necesario utilizar instrumentos diversos para identificarlos (verificando la potencia y capacidad de integración de estos instrumentos en la dinámica de clases de DCE), comprobar si son adecuados y gestionarlos visualizando los cambios y las persistencias de dichas concepciones durante el proceso de formación.

\section{Metodología de la investigación}

Metodología cualitativa; el procedimiento usado fue el de análisis de contenido de tipo semántico.

Se conjugaron instrumentos creados ad hoc con los generados en la propia dinámica de la materia (portafolios, relatos, fotos, actividades individuales y grupales, etc.). Teniendo presente que las concepciones declarativas acostumbran a ser más innovadoras que las implícitas, se seleccionaron instrumentos que recogiesen ambas.

\section{Conclusiones}

La investigación mostró que existen concepciones (sobre ciencias, métodos, paradigma de aprendizaje, roles de alumnado y profesorado, evaluación, actividades, recursos, etc.) en el profesorado en formación inicial que interfieren en la aplicación de modelos metodológicos necesarios para desarrollar competencias en general y tecnocientíficas en particular. Algunas de ellas son:

- Creencia de que para enseñar basta con saber la asignatura y aprender a enseñar no es necesario.

- Valoración de contenidos conceptuales como fundamentales y casi exclusivos. Mantenimiento para las ciencias de un fin más cultural que centrado en la formación de una ciudadanía capaz de actuar para construir un mundo sostenible y justo.

- Visión de que para enseñar ciencias son necesarias «fases teóricas» («explicación» del profesorado) previas a «fases prácticas» (realización de ejercicios, experimentos, etc.).

- Concepción del alumnado como una «caja vacía» que aprende con la explicación del profesorado.

- Suposición de que tiempo de enseñanza y tiempo de aprendizaje coinciden. Y que, con una enseñanza adecuada, todo el alumnado aprende al mismo ritmo.
- Relevancia del rol transmisivo del profesorado.

- Creencia de que el protagonismo del alumnado disminuye la eficacia del proceso.

- No significatividad de la organización espaciotemporal, que le resulta invisible y transparente.

- Referencia constante a mitos (el alumnado aprende mejor con horarios fragmentados por áreas, si se le hacen exámenes, etc., necesidad de cubrir el "programa» o trabajar todos «los temas» del libro de texto, enseñar ciencias es explicar conceptos, etc.).

- Sobrevaloración del libro de texto, guía casi única y fundamental del trabajo.

- Reduccionismo en la evaluación tanto en su finalidad como en los instrumentos utilizados.

Se observaron evoluciones y resistencias en sus concepciones después del proceso de aprendizaje. Las resistencias principales se relacionaron con dudas sobre la eficacia de la planificación conjunta profesoradoalumnado, el mantenimiento de una visión fragmentaria del currículo, la relegación de actividades como las salidas didácticas y los experimentos a meras ilustraciones a la que consideran fuente principal de información (explicación del profesorado).

Los instrumentos relacionados con relatos de aula, fotos, portafolios y actividades elaboradas durante las clases fueron los más adecuados y potentes para analizar las concepciones previas del profesorado en formación inicial.

La principal implicación de la investigación para el aprendizaje de las ciencias es la necesidad de hacer consciente al profesorado en formación inicial de sus concepciones y de acometer su reestructuración, teniendo en cuenta que algunas de ellas parecen estar interrelacionadas, con lo que su modificación resulta compleja, por lo que se requieren actuaciones integradas que permitan vivenciar en la formación inicial de DCE modelos innovadores constructivistas. 


\title{
CARACTERIZACIÓN Y EVOLUCIÓN DE LOS MODELOS DE ENSEÑAN- ZA DE LA ARGUMENTACIÓN EN CLASE DE CIENCIAS EN LA EDUCA- CIÓN PRIMARIA
}

\author{
Autor: Francisco Javier Ruiz Ortega \\ Directores: Conxita Márquez Bargalló y Óscar E. Tamayo Alzate \\ Facultat de Ciències de la Educació \\ Departament de Didàctica de la Matemàtica i de les Ciències Experimentals \\ Universitat Autònoma de Barcelona \\ Fecha de presentación: 29 de octubre del 2012
}

Palabras clave: argumentación, enseñanza de las ciencias, modelos de enseñanza de la argumentación en ciencias, pensamiento docente.

La tesis fue realizada dentro del proyecto "Desarrollo de competencias científicas: modelos y evidencias en la lectura, escritura y la experimentación", con referencia EDU2009-13890-C02-02, financiado por la Dirección General de Investigación, Ministerio de Educación y Ciencia, y el patrocinio de la Universidad de Caldas, Manizales-Colombia.

\section{Resumen}

En la investigación participaron cinco docentes de educación primaria de la institución educativa $\mathrm{Fe}$ y Alegría de la ciudad de Manizales, Colombia, que imparten clases a niños y niñas cuyas edades están entre los 9 y 10 años; dos de los docentes fueron elegidos para realizar el estudio de caso.

La investigación tiene tres propósitos: a) caracterizar y comprender los cambios en el perfil del pensamiento sobre la argumentación y en la capacidad argumentativa de un grupo de cinco docentes, $b$ ) identificar los modelos de enseñanza de la argumentación en el aula de ciencias de los docentes que participan en el proceso de reflexión crítica y $c$ ) comprender la evolución de los modelos de enseñanza de la argumentación identificados en los docentes.

En el aspecto teórico destaca la discusión sobre cuatro aspectos: a) la argumentación como competencia científica, $b$ ) la argumentación desde la teoría de Toulmin y desde las adaptaciones que a esta teoría se han realizado en el campo de la didáctica de las ciencias, c) la importancia que tiene para la enseñanza de las ciencias implicar a los docentes en procesos de reflexión y $d$ ) la integración de los perfiles de pensamiento y de desempeño de los docentes para identificar y caracterizar los modelos de enseñanza de la argumentación en ciencias.
En el aspecto metodológico, la investigación se sitúa dentro del paradigma cualitativo y destacan elementos complementarios de la etnografía, la investigación acción y los diseños de estudio de caso para la comprensión en profundidad de los fenómenos. La información se obtiene a partir de distintas fuentes: $a$ ) un cuestionario constituido por preguntas abiertas y que se aplica antes y después del proceso de reflexión crítica (proceso que duró siete meses), $b$ ) las programaciones y c) los registros en vídeo y en audio de las clases, las entrevistas y los encuentros de reflexión crítica realizados con los docentes.

El seguimiento riguroso del proceso y el análisis de contenido y de discurso realizado a la información obtenida permitieron caracterizar los perfiles de pensamiento y de desempeño de los docentes, perfiles que tras su integración se constituyeron en la base para la comprensión de la evolución de los tres modelos de enseñanza de la argumentación en ciencias.

Los resultados indican cómo la crítica a los aspectos de orden: a) epistemológico, específicamente a la perspectiva tradicional de la construcción de la ciencia; $b$ ) didáctico, concretamente al rol autoritario del docente, a la ciencia de carácter dogmático que se imparte en el aula y a las interacciones comunicativas autoritarias entre docente y estudiantes, y $c$ ) conceptual, particularmente a la aceptación de la argumentación como una acción para dar información basada en datos y afirmaciones, potenció cambios en el desempeño de los docentes, fundamentales para promover la evolución gradual de los modelos de enseñanza de la argumentación.

Finalmente, esta investigación sugiere algunas implicaciones que contribuyen a su fortalecimiento y también al inicio de nuevas propuestas de investigación, como por ejemplo el estudio de las acciones argumentativas de los estudiantes en el aula de ciencias durante la educación primaria o la evaluación de las aportaciones de los libros de texto a los procesos argumentativos. 


\title{
APORTACIONES DE LA ENSEÑANZA CON ANALOGÍAS AL DESARRO- LLO DEL PENSAMIENTO MODELIZADOR DE LOS ALUMNOS ACER- CA DEL CAMBIO QUÍMICO
}

\author{
Autora: María del Mar Aragón Méndez \\ Directores: José María Oliva Martínez y Antonio Navarrete Salvador \\ Programa de doctorado: Investigación y Prácticas educativas \\ Universidad de Cádiz \\ Departamento de Didáctica \\ Fecha de presentación: 2 de febrero del 2012
}

La enseñanza y el aprendizaje de las ciencias en el siglo XXI se enfrenta a nuevos retos: no basta con aprender ciencias; las nuevas necesidades sociales hacen necesario aprender a hacer ciencias y aprender sobre las ciencias. En esta línea, cobra una especial relevancia la enseñanza y el aprendizaje en torno a los modelos científicos, precisándose recursos que ayuden a los alumnos a ser artífices de su propio aprendizaje, de modo que sean capaces de aprender modelos, aplicarlos, revisarlos y reconstruirlos. Las analogías se presentan como un instrumento sumamente prometedor al respecto, pudiendo contribuir al aprendizaje de conceptos y de procedimientos, así como de valores, visiones y compromisos epistemológicos coherentes con el proceso de construcción de la ciencia y con su naturaleza. Esta perspectiva, orientada al aprendizaje del cambio químico, da pie a la cuestión central que origina la investigación: ¿En qué medida, y de qué forma, puede contribuir el aprendizaje mediante analogías al desarrollo del pensamiento modelizador de los alumnos en torno al cambio químico?

Para responder a esta pregunta, y mediante un enfoque fundamentalmente interpretativo, se estudian varias dimensiones del aprendizaje de los alumnos tras la implementación de una propuesta didáctica que tiene como eje vertebrador la modelización en el ámbito del cambio químico y en la que se utilizan como herramienta recurrente actividades de carácter analógico.

Las dimensiones del aprendizaje analizadas en la investigación son:

- La evolución de los modelos explicativos de los alumnos sobre el cambio químico. Se ha estudiado la progresión en el uso de modelos proposicionales o verbales -referidos tanto a la interpretación a escala macroscópica como submicroscópica del cambio químico-, así como en el uso de modelos simbólicos de carácter modal (empleando diagramas pictóricos de partículas) y amodal (representación mediante símbolos de elementos, fórmulas químicas y ecuaciones).

- Las capacidades desarrolladas que se relacionan con la modelización en ciencias, entendida esta en el sentido más amplio; esto es, las habilidades relacionadas con el aprendizaje de modelos, con el desarrollo las destrezas necesarias en el proceso de modelización (aplicación, revisión y reconstrucción de modelos) y con el aprendizaje sobre la naturaleza de los modelos.

- Las capacidades relativas al pensamiento analógico. En concreto, y paralelamente al análisis de las competencias relacionadas con la modelización, se ha estudiado en qué medida los estudiantes han aprendido analogías, han aprendido a aplicarlas, a revisarlas, a participar en su (re)construcción y a crearlas, y si han aprendido sobre la propia naturaleza de las analogías.

- La vinculación existente entre el desarrollo de la competencia de modelización y la competencia analógica.

Los resultados del análisis muestran, en primer lugar, que las actividades analógicas parecen desempeñar un papel importante en la evolución de los modelos explicativos de los alumnos sobre el cambio químico. El aprendizaje es especialmente satisfactorio en el empleo del modelo icónico modal, que es fundamentalmente un modelo analógico. Además, las interpretaciones verbales del cambio químico a escala submicroscópica se encuentran especialmente vinculadas al uso del modelo icónico.

En segundo lugar, una parte importante de los alumnos han aprendido, al menos a un nivel básico, a aplicar modelos, a revisarlos y a reconstruirlos, desarrollando no solo habilidades de carácter procedimental directamente relacionadas con el proceso de modelización, sino también visiones epistemológicas en 
torno a la naturaleza de los modelos. Las capacidades que se muestran más complejas son aquellas relacionadas con el proceso de modelización que requieren una mayor dosis de imaginación y creatividad.

En tercer lugar, y respecto al desarrollo del pensamiento analógico, podemos afirmar que los alumnos, en la mayoría de los casos de forma satisfactoria, no solo aprendieron analogías, sino que también aprendieron a usarlas. Aprendieron, además, aunque a un nivel ligeramente inferior, a revisarlas de forma adecuada y, en menor medida, a reconstruir analogías. Al trabajar con analogías, los alumnos desarrollaron capacidades relacionadas con la comprensión de la naturaleza de las analogías y con los procedimientos que implican el trabajo con estas. Las capacidades que requerían cierta creatividad, imaginación y reflexión se potenciaron a través del trabajo colaborativo.
En cuarto lugar, y refiriéndonos a la vinculación entre modelización y pensamiento analógico, el empleo de actividades analógicas en el contexto del cambio químico parece favorecer el aprendizaje de conceptos, de modelos y de los procedimientos acordes a la metodología propia de la ciencia que se ponen en juego en la modelización. También podemos suponer una contribución del pensamiento analógico al desarrollo de la imaginación, de la creatividad y de aptitudes y actitudes necesarias para el uso crítico de los modelos científicos. En cualquier caso, todo apunta a que los mecanismos que se ponen en juego cuando los alumnos trabajan con analogías tienen mucho en común con los necesarios en el proceso de modelización, pudiéndose favorecer mutuamente y existiendo un gran paralelismo entre el desarrollo del pensamiento modelizador y del pensamiento analógico.

\title{
TEORÍA, ESTRUCTURA Y MODELOS ATÓMICOS EN LOS LIBROS DE TEXTO DE QUÍMICA DE EDUCACIÓN SECUNDARIA. ANÁLISIS DES- DE LA SOCIOLOGÍA DE LA CIENCIA E IMPLICACIONES DIDÁCTICAS
}

\author{
Autora: Diana María Farías Camero \\ Tesis doctoral \\ Director: Dr. Josep Castelló Escandell \\ Departamento de Didáctica de las Ciencias Experimentales y la Matemática, \\ Universidad de Barcelona \\ Programa de Doctorado en Formación del Profesorado: Práctica educativa y comunicación \\ Fecha de la presentación: 15 de octubre del 2012
}

\section{Resumen}

En esta tesis se aporta un análisis de los contenidos relacionados con el modo como se presenta la ciencia en los libros de texto escolares desde una perspectiva sociológica de las ciencias. Se desarrolla y aplica una metodología en la que se emplean elementos del trabajo del sociólogo de la ciencia Bruno Latour para analizar una muestra de libros de texto escolares de Química de educación secundaria publicados en territorio español desde 1845 hasta el 2010. Las bases teóricas que sustentan el trabajo son el enfoque de aportaciones de la historia y la filosofía de la ciencia en la enseñanza de las ciencias (HFC) y su línea de investigación en naturaleza de la ciencia $(\mathrm{NdeC})$, la sociología de las ciencias, los libros de texto y las investigaciones en di- dáctica de las ciencias referentes al tema central de esta tesis: teoría, estructura y modelos atómicos. La revisión teórica se ha desarrollado con un enfoque historicista en el que lo relevante es mostrar las diferentes transformaciones y la evolución de los cuatro tópicos mencionados, con el fin de ubicar al lector en un escenario en el que se sustenta cómo la sociología de las ciencias ha sido excluida de la investigación en didáctica de las ciencias, pero en el que, a la vez, se señalan las condiciones que posibilitan aportaciones desde la sociología, tanto desde lo teórico como desde lo metodológico, para el análisis de cómo se construye el conocimiento científico en los libros de texto escolares de ciencias y cómo esas aportaciones pueden inscribirse dentro del enfoque HFC. 
El marco metodológico, de carácter ampliamente cualitativo y descriptivo, apuesta por la complejización, entendida como la posibilidad de combinar el análisis de algunos aspectos que dan cuenta de la ciencia y la práctica científica desde lo epistemológico, la evaluación de los eventos temporales del pasado y una revisión de las imágenes, que se ponen en común con el método propuesto en este trabajo, en el que los elementos del modelo de la circulación de la ciencia, los humanos y los no-humanos reflejan la presencia de redes dentro de los libros de texto que muestran la manera como circula la ciencia. En el método desarrollado, se entienden los diferentes actores que participan para describir cómo se mueve la ciencia desde su epicentro hasta la esfera de lo público, a modo de nodos que pueden estar conectados en mayor o menor grado dependiendo de cómo los autores de los libros de texto entienden y reflejan qué son la ciencia y el trabajo científico.
Los resultados se presentan a través de esas cuatro perspectivas: una que, desde el modelo de circulación de la ciencia y la teoría de redes, permite evidenciar cómo se habla de la ciencia, de los científicos y de la práctica científica en los libros de texto. Otra que da cuenta de las creencias epistemológicas de los autores de los libros con respecto a diferentes aspectos de la ciencia y la práctica científica; otra en la que se analizan las referencias temporales como indicio de la importancia de la contextualización histórica de los contenidos analizados, y una última que revisa el manejo de las imágenes alrededor de los principales actores en el relato científico escolar. Las conclusiones se articulan alrededor de las relaciones HFC-NdeC y sociología de las ciencias, la evaluación de los libros de texto y la metodología desarrollada, los contenidos que hablan sobre la ciencia en los libros de texto y la imagen de ciencia que transmiten, sobre los libros de texto como actores en la red de la ciencia y sobre los profesores que usan los libros. 